\title{
Performance of the ATLAS Resistive Plate Chambers
}

\section{Workshop on Resistive Plate Chambers and related detectors}

GSI - Darmstadt February $9-12,2010$

Giordano Cattani

University of Rome "Tor Vergata" \& INFN Roma 2 on behalf of the ATLAS Muon Collaboration 


\section{Overview}

- Introduction

- The ATLAS experiment

- The ATLAS Muon Spectrometer

- Resistive Plate Chambers (RPCs) of the ATLAS experiment

- RPCs performance with 2009 cosmics data

- Cluster size

- Spatial resolution

- Efficiency 


\section{A Toroidal LHC ApparatuS (ATLAS)}

$\rightarrow \quad$ Inner Detector: $6 \mathrm{~m}$ long $1.1 \mathrm{~m}$ radius inside $2 \mathrm{~T}$ Solenoid

$\rightarrow \quad$ Pixels

$\rightarrow \quad$ SCT Silicon Strips

$\downarrow \quad$ TRT Transition Radiation Tracker e/ $\pi$ separation

Calorimetry $\mid \eta \overline{\mid}<4 . \overline{9}$

$\downarrow \quad$ EMBC, EMEC accordion LAr $+\mathrm{Pb}|\eta|<3.2$

$\rightarrow \quad$ Tile Hadronic Fe + scintillator $|\eta|<1.7$

$\diamond \quad$ HEC Hadr end cap Cu+Lar $1.5<|\eta|<3.2$

$\downarrow \quad$ FCAL Forward calo Cu+W+Lar $3.1<|\eta|<4.9$

EM energy resolution

- $\sigma(E) / E=10 \% / \sqrt{ } E \oplus 0.7 \%$

Hadronic energy resolution (jets)

- $\sigma(E) / E=50 \% / \sqrt{ } E \oplus 3 \% \quad(|\eta|<3.2)$

- $\sigma(E) / E=100 \% / \sqrt{ } E \oplus 10 \% \quad(|\eta|>3.2)$

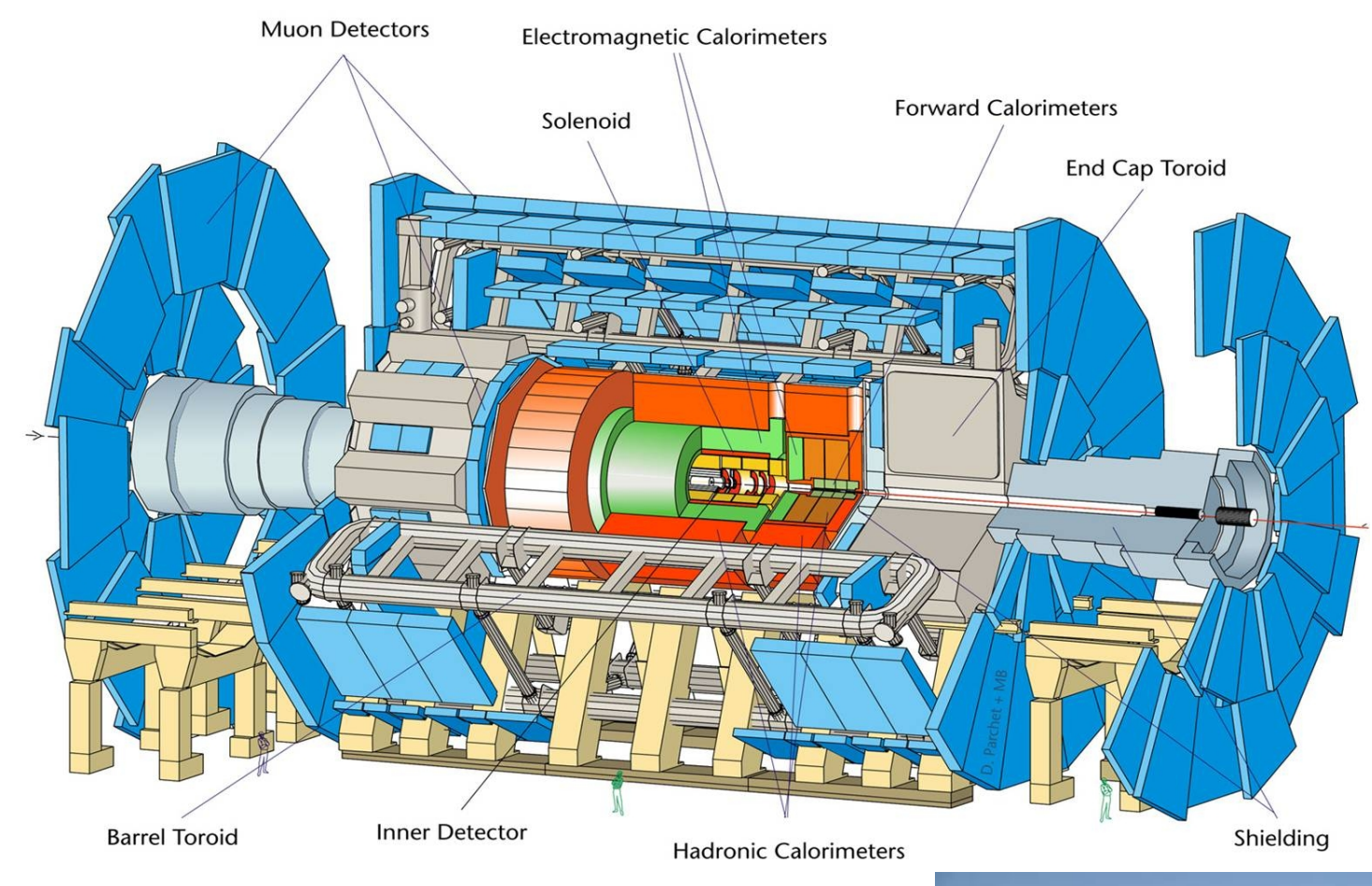

\begin{tabular}{l|l|l}
\hline Diameter & $25 \mathrm{~m}$ & \\
\hline $\begin{array}{l}\text { Barrel region lenght } \\
\text { Distance between Big- }\end{array}$ & $\mathbf{2 6} \mathrm{m}$ & \\
Wheels & $7000 \mathrm{t}$ & The same as Eiffel Tower \\
Mass & & \\
\end{tabular}

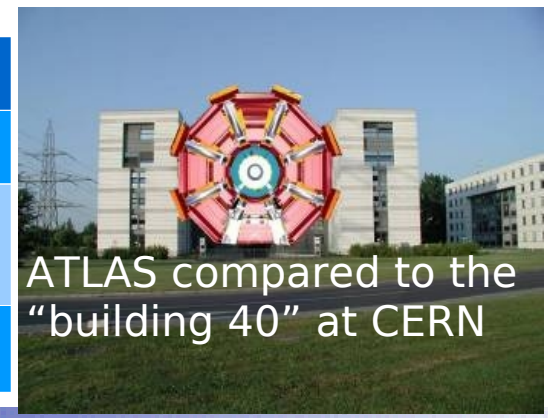

G.Cattani - giordano.cattani@cern.ch

INFN

University of Rome "Tor Vergata" \& INFN Roma 2

$\mathrm{B}^{\mathrm{A}}$

February 9, 2010 / Page 3

$X$ Workshop on Resistive Plate Chambers and related detectors 


\section{Muon Spectrometer: geometry in the barrel region}

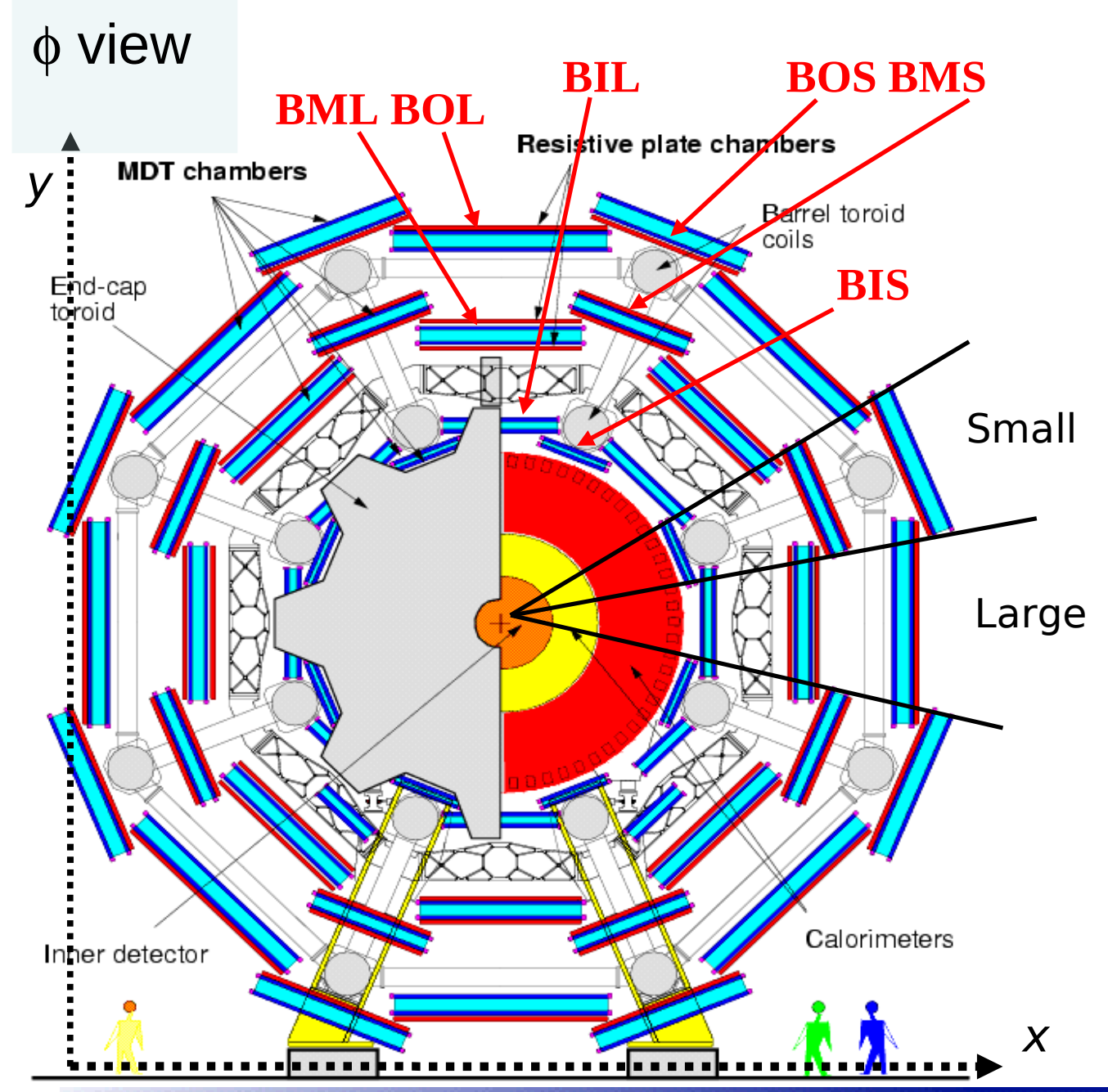

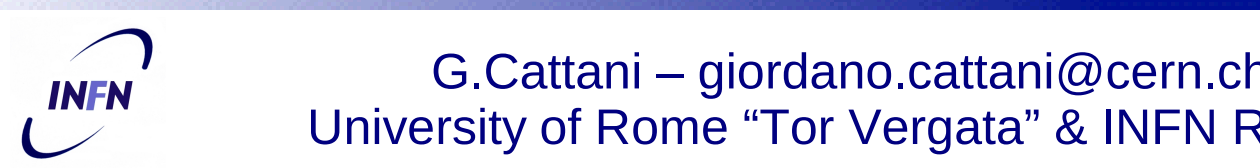

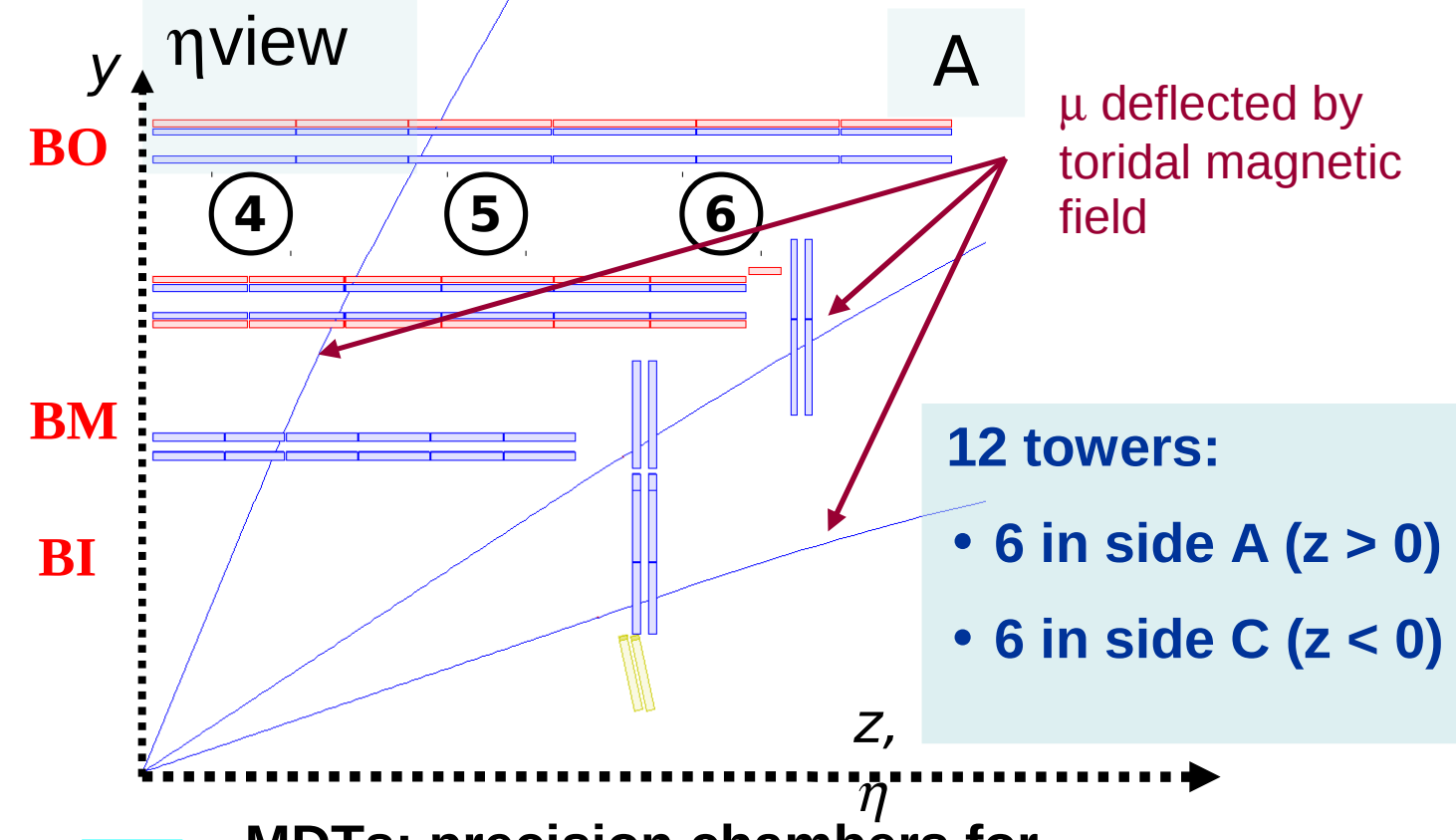

MDTs: precision chambers for tracking in $\eta$ view - $35 \mu \mathrm{m}(|\eta|<2.7)$

RPCs: trigger LVL1 + second coordinate measurement $(|\eta|<1.05)$ In the foreward region TGC chambers are used to trigger $(|\eta|<2.4)$ and measure the second coordinate $(1.05<|\eta|<2.7)$
February 9, 2010 / Page 4 $X$ Workshop on Resistive Plate Chambers and related detectors 


\section{Resistive Plate Chambers (RPCs) of the ATLAS experiment}

- Spatial and time resolution: $10 \mathrm{~mm} \times 1 \mathrm{~ns}$

- High rate capability up to $\sim 1 \mathrm{kHz} / \mathrm{cm}^{2}$

- Tested for aging up tp $100 \mathrm{~Hz} / \mathrm{cm}^{2}$ per 10 ATLAS equivalent years with a safety factor of 5

- Used as trigger chambers of the ATLAS LVL1 trigger system

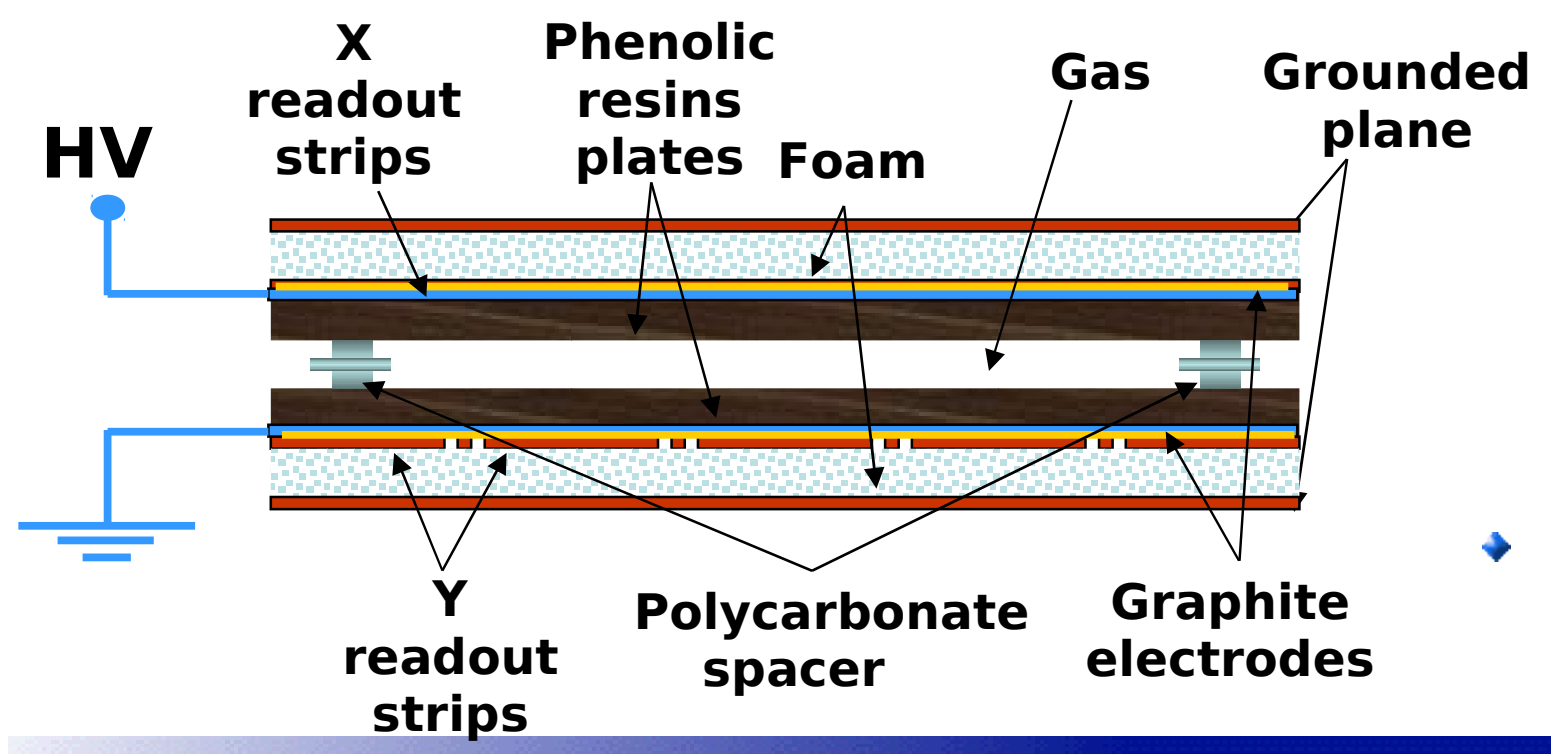

$C^{\text {INFN }}$

G.Cattani - giordano.cattani@cern.ch $\rightarrow \mathrm{HV} \sim 9.6 \mathrm{kV}\left(@ 22{ }^{\circ} \mathrm{C}\right.$ and p = 970 mbar)

- Electrode plate resistivity $\sim 10^{10} \Omega \mathrm{cm}$

- Gap width $=2 \mathrm{~mm}$

- Gap internal surfaces treated with linseed oil

- Copper read-out strips for orthogonal coordinates measurement applied on both sides of the gap. Typical strip pitch $\sim 30 \mathrm{~mm}$

- A group of strips measuring the same coordinate is defined as a read-out panel.

- Gas mixture: $\mathrm{C}_{2} \mathrm{H}_{2} \mathrm{~F}_{4} 94.7 \%-\mathrm{C}_{4} \mathrm{H}_{10} 5 \%-\mathrm{SF}_{6} 0.3 \%$

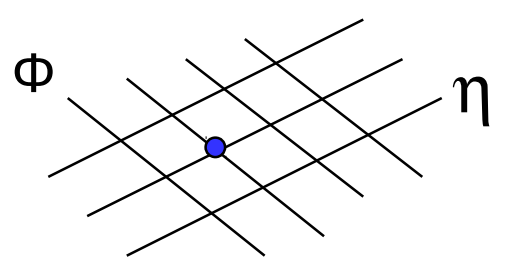

RPCs measure the coordinate orthogonal to the bending direction and therefore can reconstruct a track in 3-dimensions

Chambers and related detectors 


\section{RPC calibration /1}

- The ATLAS detector is fully installed in the cavern and an intense work to test the apparatus and to study in situ the detector performance is ongoing.

- RPC performance is evaluated using tracks of cosmic muons reconstructed by the precision tracking chambers in the barrel region (Monitored Drift Tube - MDT)

- This studies are important to verify that performances are as expected by design and to check integration of the chambers in the full apparatus.

- Results shown in the following slides are from data recorded over the full Muon Spectrometer ( 8000 read-out panels)

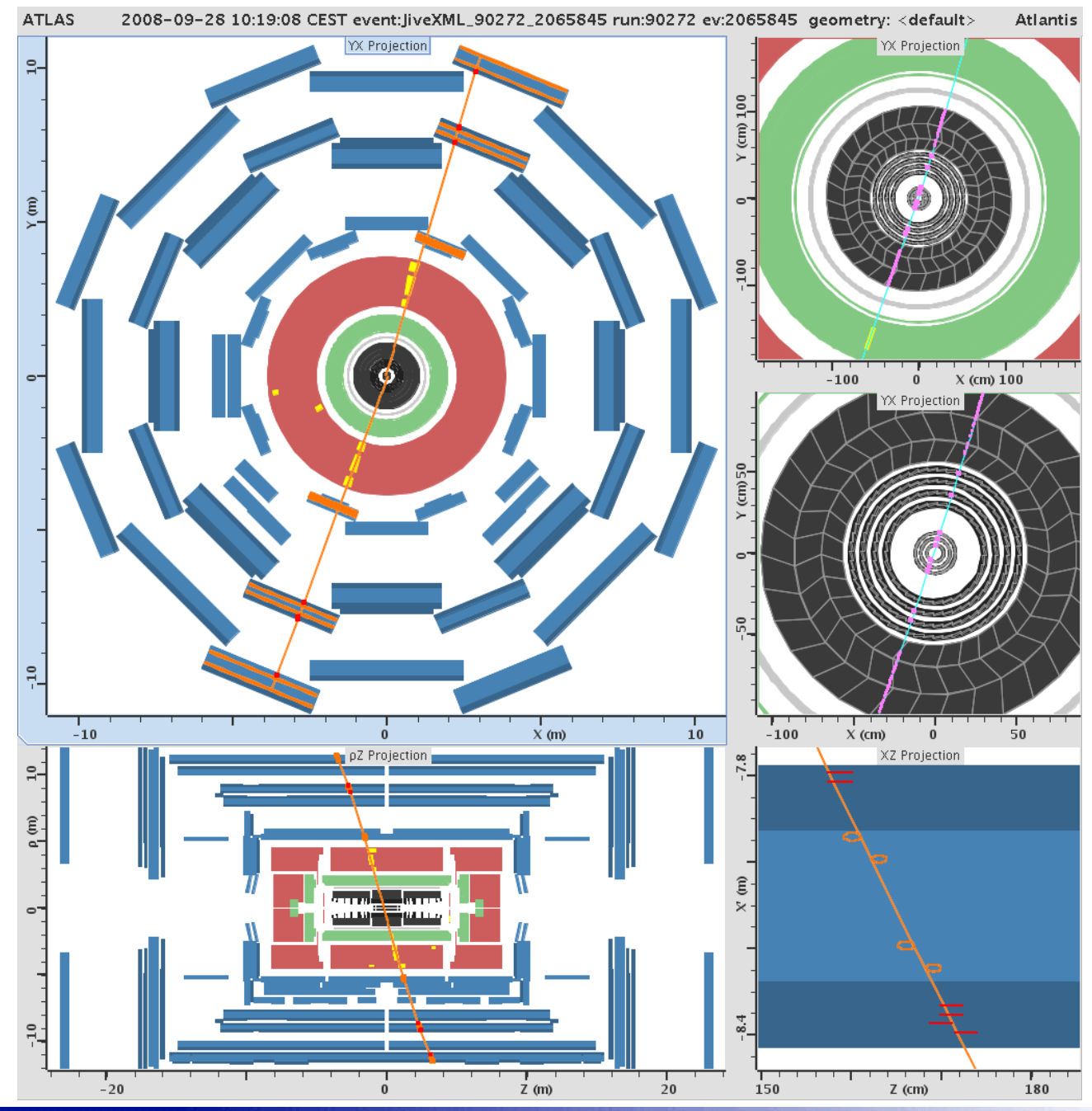




\section{RPC calibration /2}

- The RPC calibration goal is to optimize working parameters of the detector

- using dedicated high statistics data set (calibration stream) or

- normal physics data set (full stream) as a back-up solution or for specific studies

- Results stored in the official ATLAS conditions database (COOL)

- data available to reconstruction algorithms

- Use distributed computing resources

- The results of this talk have been obtained using the full stream 


\section{RPC calibration /3}

- Results include several detector-related quantities:

- Efficiency

- Cluster size (CS)

- Space residuals

- Using precise tracking from MDT chambers

- bias on measurements due the trigger is removed during off-line analysis

- extrapolation to RPC layers takes into account materials and magnetic field

- precise extrapolation allows to determine spatial resolution and to study small local effects

- Granularity up to the strip level

- Averages per panel provided as well

- If not otherwise specified each entry in the following histograms represents a panel 


\section{Results}




\section{Cluster size /1}

- Mean panel cluster size for different chambers type

- Calculated requiring 1 or 2 muon tracks in the event

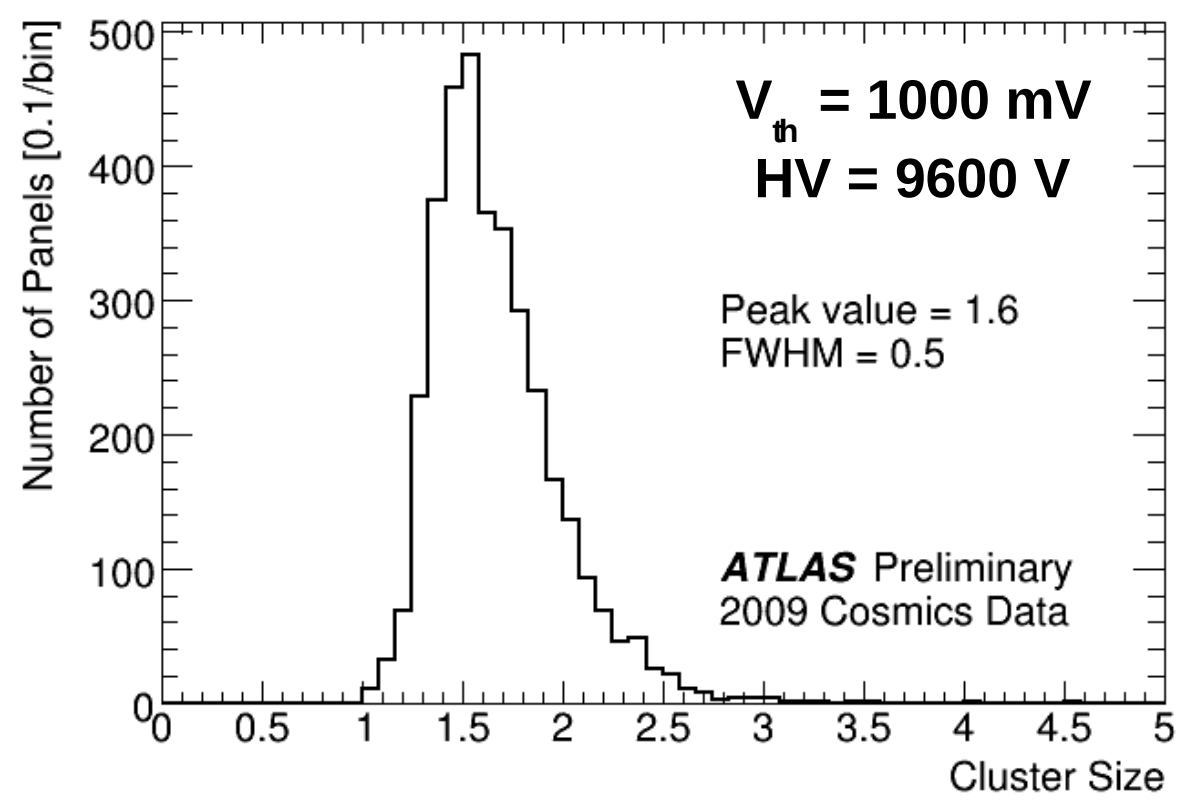

- Cluster size BM chambers

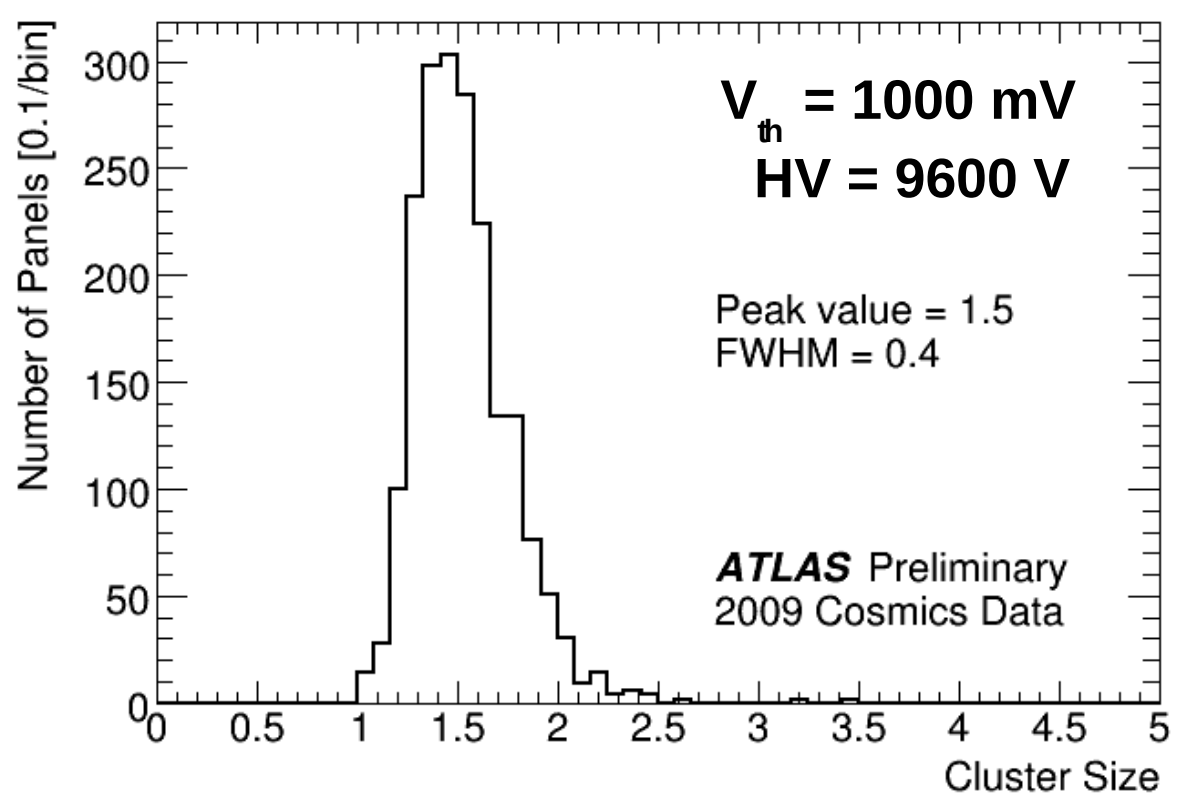

- Cluster size for BO chambers

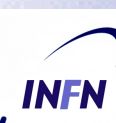

INFN $\mathcal{L}$
G.Cattani - giordano.cattani@cern.ch

University of Rome "Tor Vergata" \& INFN Roma 2
February 9, 2010 / Page 10 $X$ Workshop on Resistive Plate Chambers and related detectors 


\section{Cluster size /2}

Cluster size for different $V_{\text {th }}$ values

$$
\mathrm{HV}=9000 \mathrm{~V}
$$

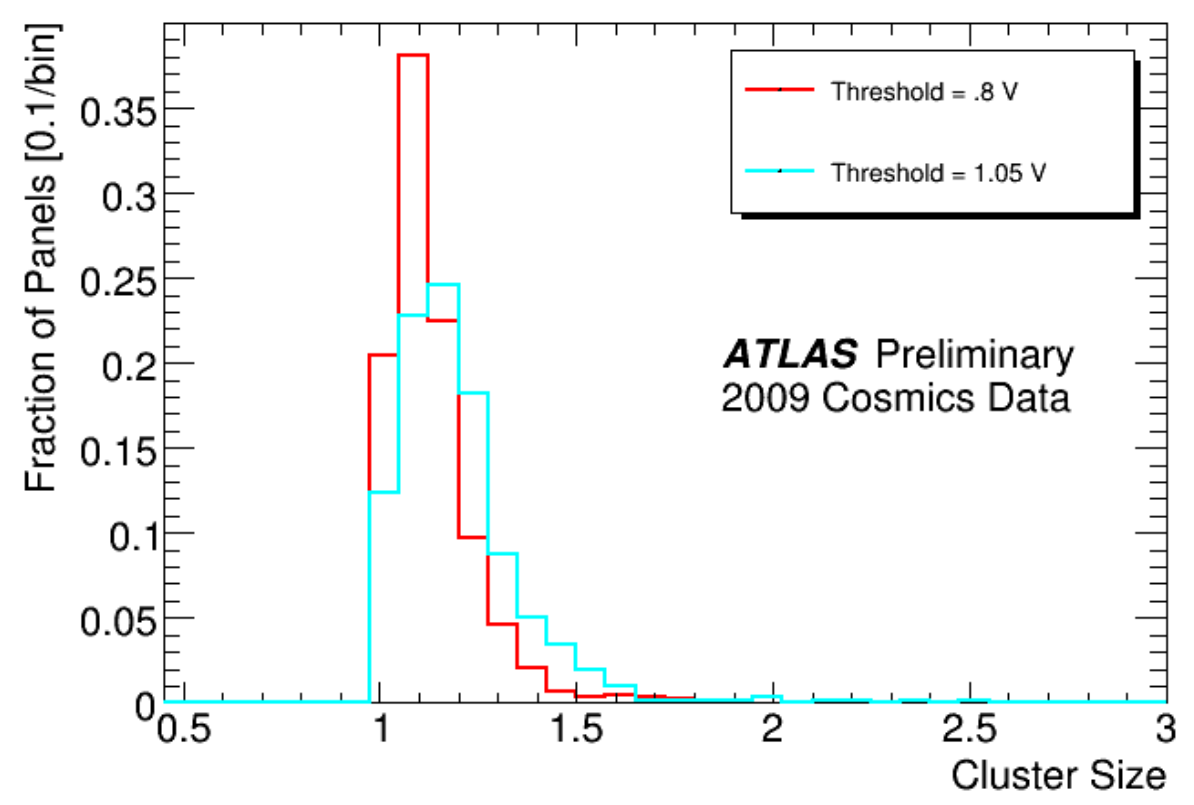

- At $9000 \mathrm{~V}$ the cluster size has only a very low dependency on $\mathrm{V}_{t h}$, as expected
Cluster size dependence on HV

$$
\mathrm{v}_{\mathrm{th}}=1000 \mathrm{mV}
$$

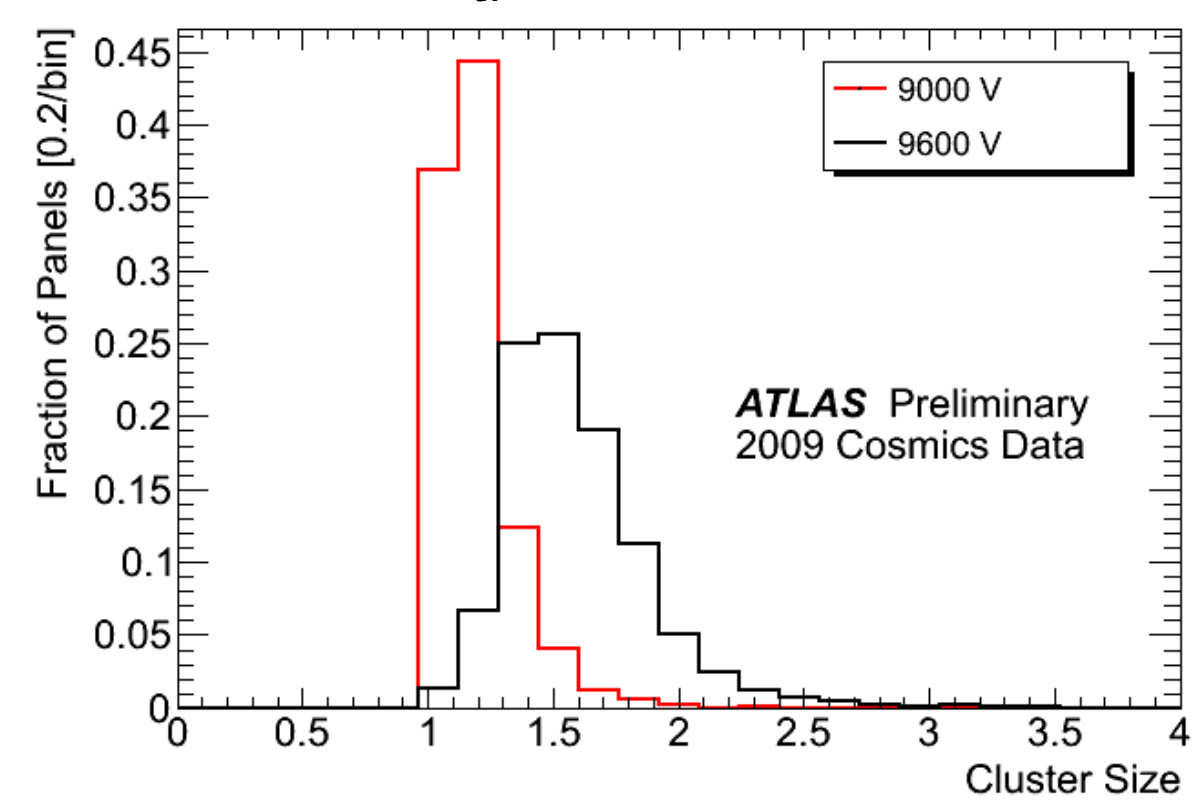

- Dependence on HV at fixed $V_{t h}$ is as expected

- NB: higer $V_{\text {th }}$ values correspond to effective looser threashold 


\section{Spatial resolution}

- Considering only $\eta$ view to exploit MDT tracking precision

- Gaussian fit of residual distribution

- Sigma from the fit = resolution

- $\quad$ Separate cluster size = 1, 2

- Residuals are normalized to strip width to ease the comparison between different chambers

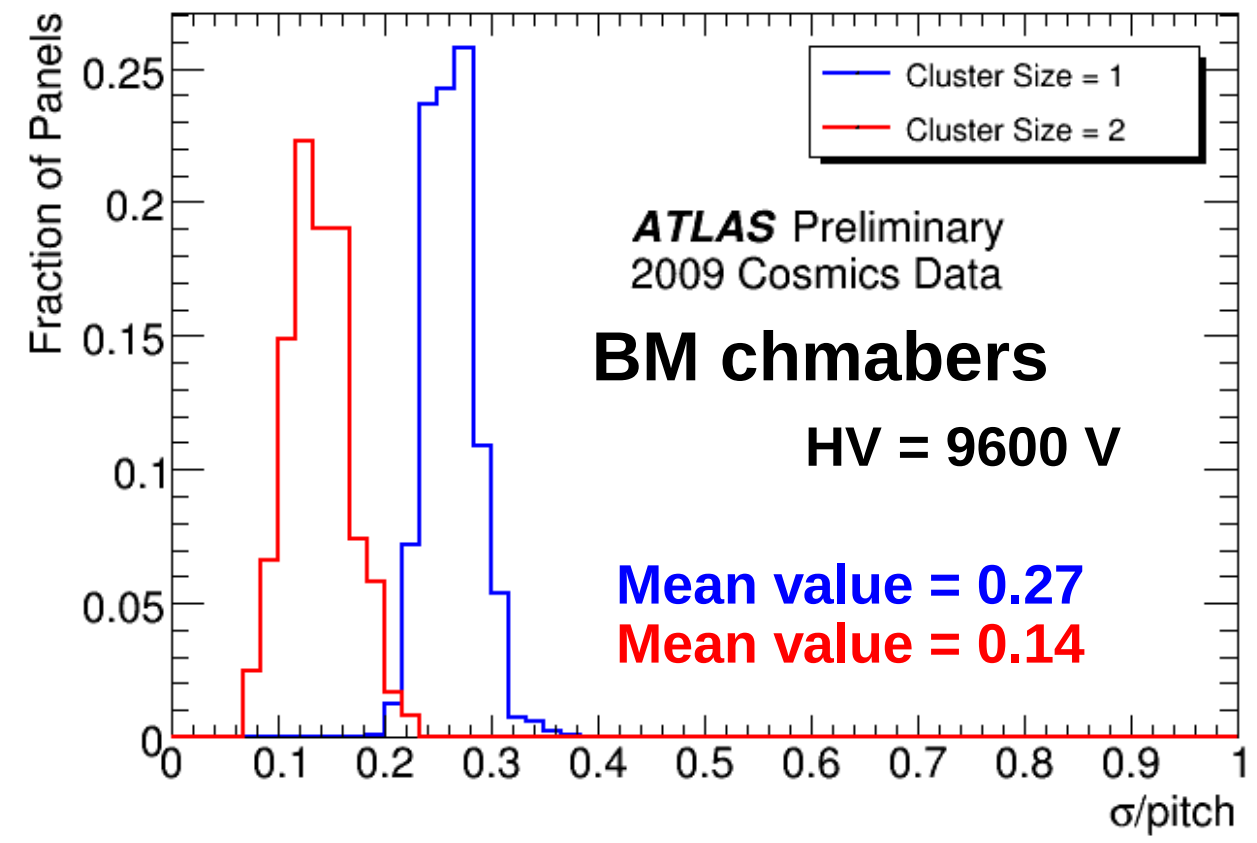

$$
\text { Residual }=z_{\text {Feader }} \quad-z_{\text {MrTradk }}
$$

- Spatial resolution normalized to strip pitch $(\sim 30 \mathrm{~mm})$ for clusters of size 1(2) in blue (red)

- Better resolution for cluster size 2 as expected 


\section{Efficiency vs sector}

- Efficiency determination algorithm:

- Extrapolate each muon track, reconstructed from MDT hits, to an RPC plane

- Check if in a region of $\pm 70 \mathrm{~mm}$ around the impact point an RPC hit is present

- Note: time acceptance window for hits is 200 ns

- The blue distribution shows panels with a panel mean hit time less than 25 ns ( 1 bunch crossing), i.e. close to the border of the time acceptance window

- this can cause loss of hits

- NB: readout timing is being tuned to eliminate this effect

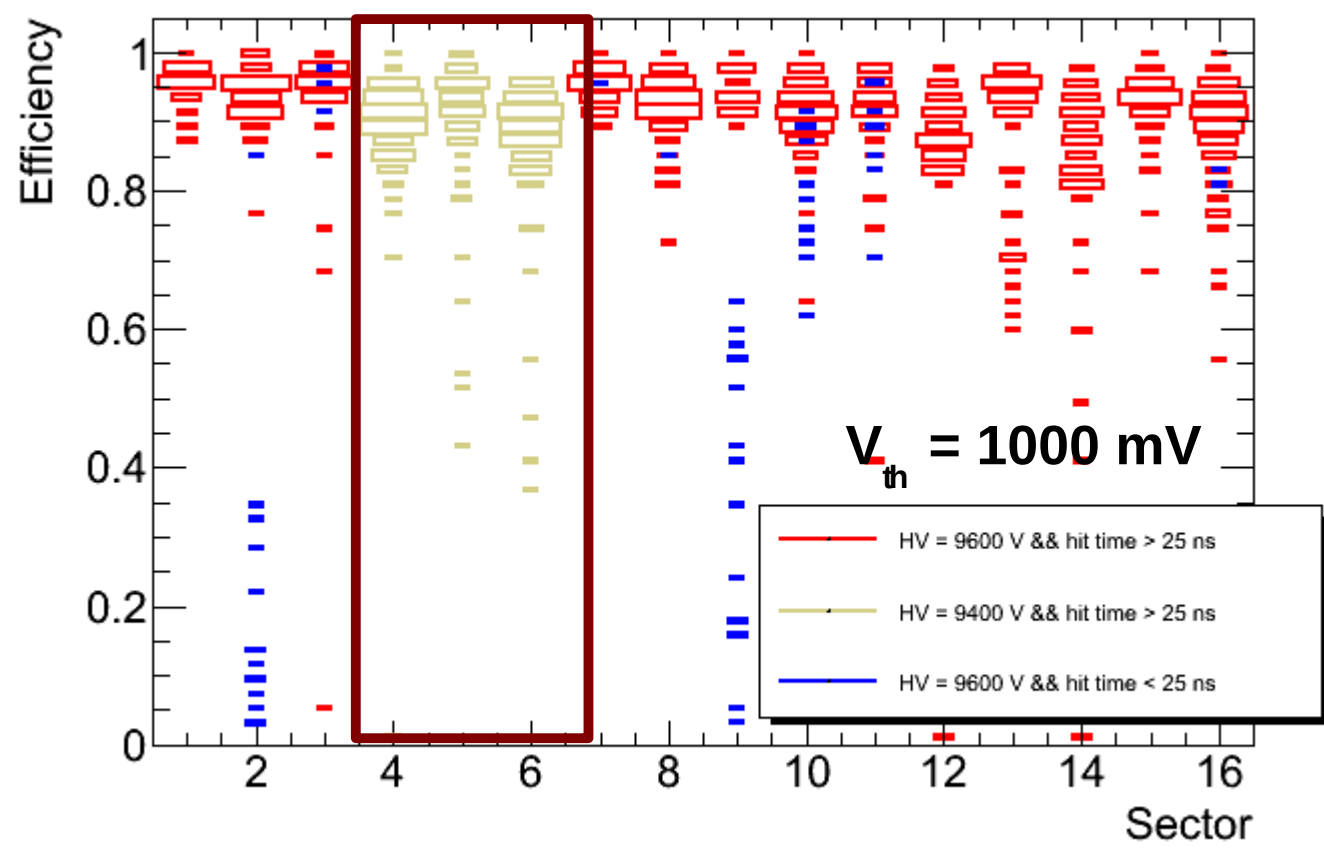

- Efficiency vs sector

- All chambers @9600 V except for sectors 4,5,6@9400 V

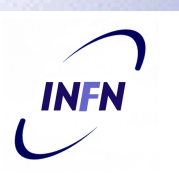

G.Cattani - giordano.cattani@cern.ch University of Rome "Tor Vergata" \& INFN Roma 2

February 9, 2010 / Page 13 $X$ Workshop on Resistive Plate Chambers and related detectors 


\section{Efficiency vs cluster size}

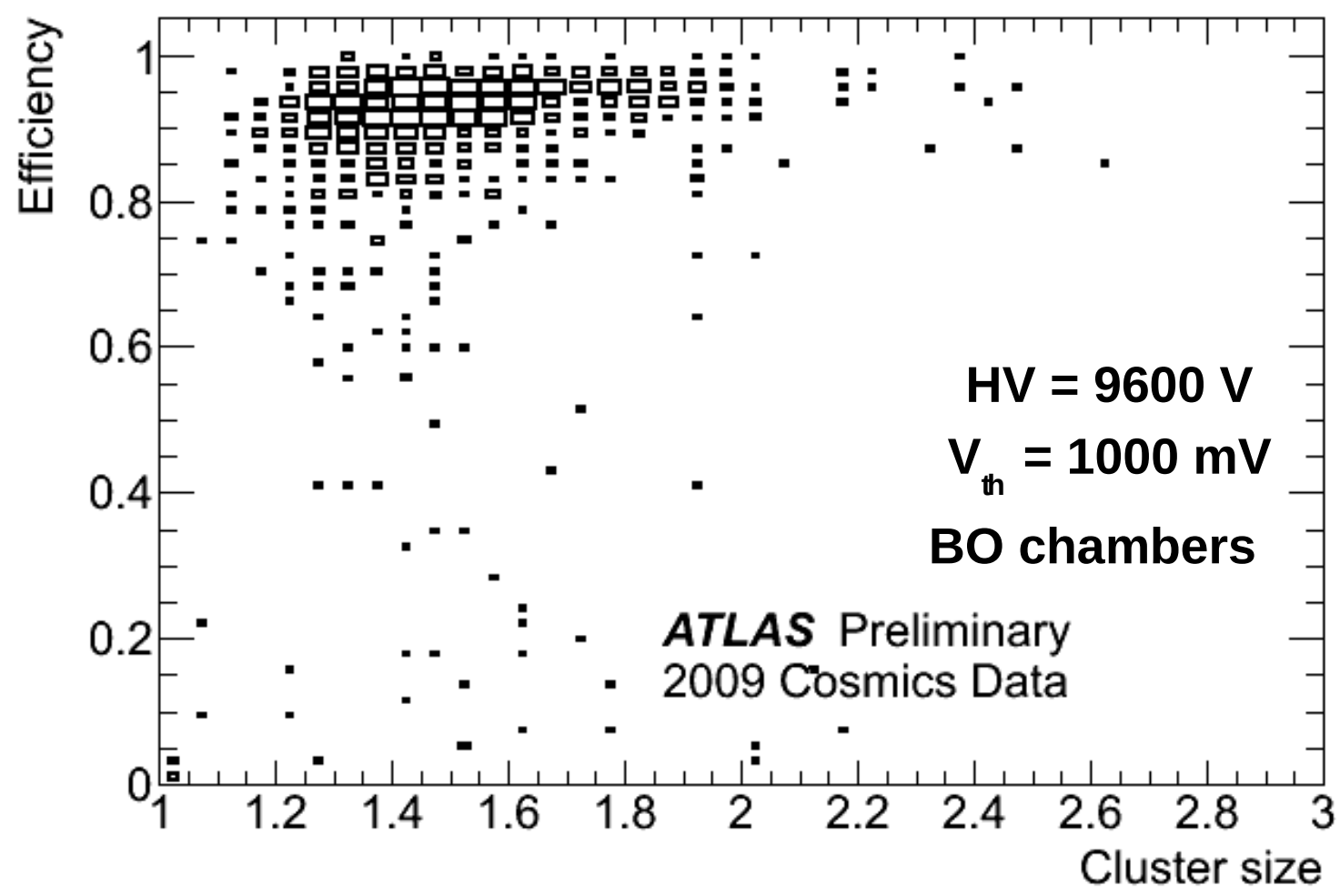

- Efficiency vs cluster size for BO chambers @9600 V

- Efficiency not corrected for pressure and temperature

- Efficiency vs cluster size behaves as expeceted

INFN




\section{Chamber alignment}

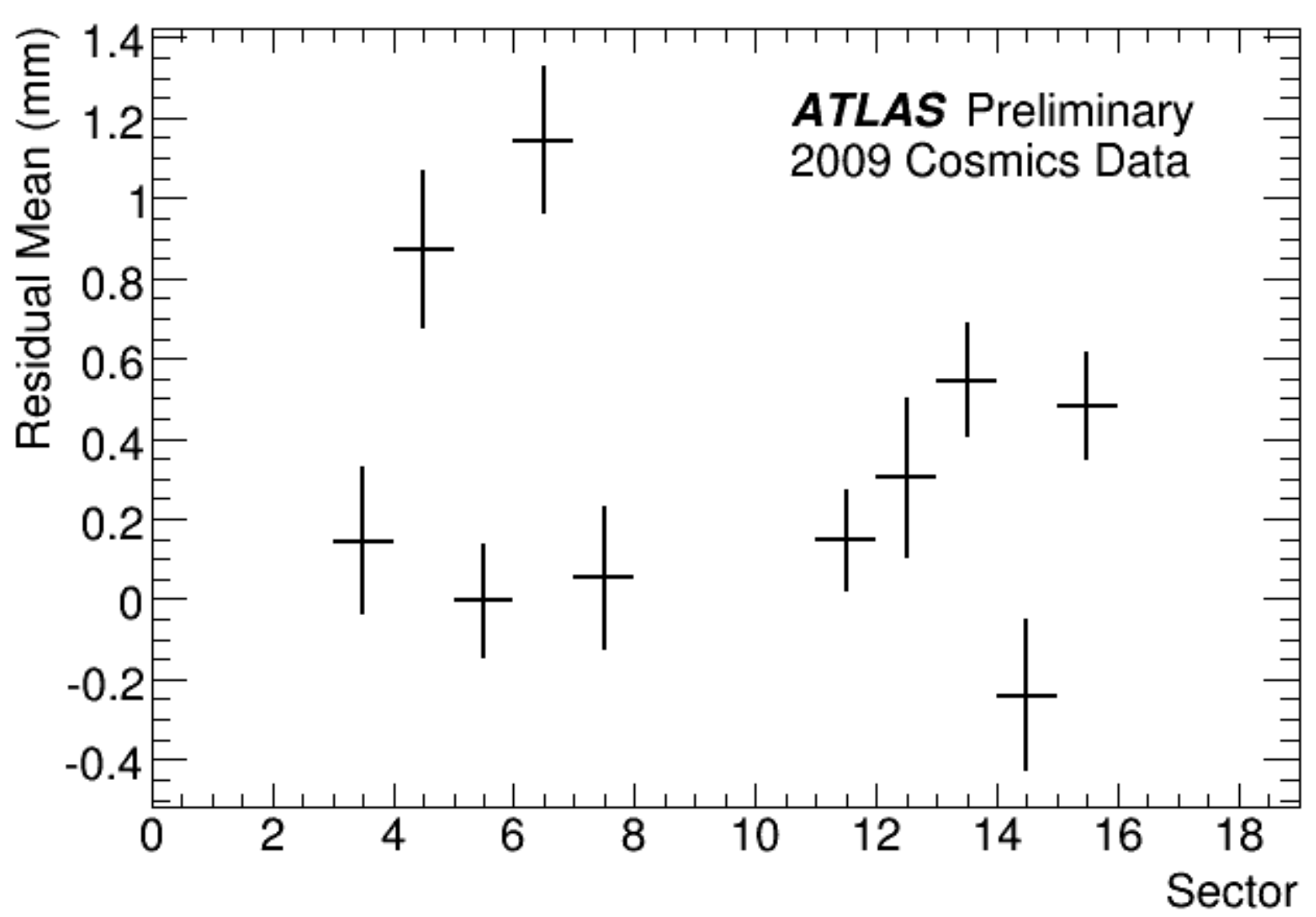

- The mean value of the residual distribution of each ETA panel is plotted for different sectors

- Displacement of RPC panels wrt MDT chambers up to fraction of millimeters is detectable thanks to the very MDT high tracking precision

- Sectors 1, 2, 8, 9, 10,16 are missing due to low statistics (vertical sectors) 


\section{Tools}




\section{Muon calibration stream}

- A detailed and reliable measurement of the detector response, up to the level of the individual strip, needs a significant number of muon tracks to be analyzed

- A dedicated stream has been foreseen to answer to this necessities, called muon calibration stream.

- It contains the output of the Level 1 muon trigger (before any Level 2 selection).

- It comes at a much higher rate $(\sim 1 \mathrm{kHz})$, i.e. much higer statistics, than the events selected by the full trigger chain with a rate $(\sim 100 \mathrm{~Hz})$

- Each muon calibration stream event contains only hits from the muon spectrometer, in a region where a muon trigger occurred

- The analysis is splitted in two separate steps

- Creation of dedicated RPC calibration ntuples

- Processing of the ntuples to obtain calibration results 


\section{The use of the GRID}

- In order to gain time, both steps need to be parallelized

- Ntuple production via the GRID

- Automatic splitting: for example, one job per input file

- Ntuples registered on the GRID (in a dataset) for later access

- Calibration results obtained through ROOT macro

- Input is one or more file from datasets obtained in previous steps

- Output is text file in a custom format with a summary of all relevant information

- Written to a dataset as well, and output from several jobs can be easily merged

- The merging step is where one can choose how many runs to group together 


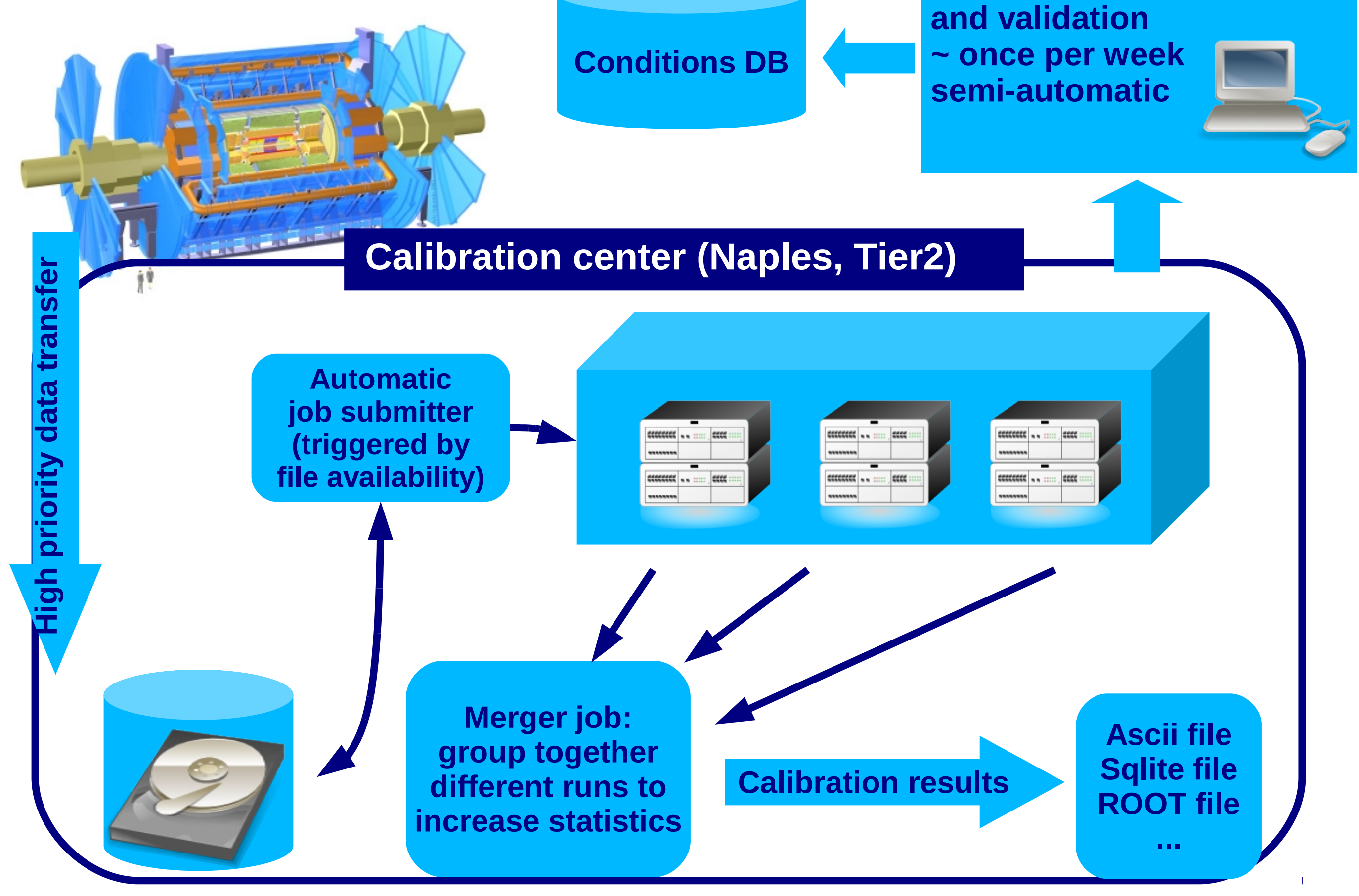




\section{Conclusions}

- The ATLAS RPC system is a very complex setup, which will allow to perform large scale studies of the detector performance with unprecedented sensitivity

- An extensive set of measurements has been already done using cosmic rays

- Preliminary results shown here are in agreement with expectations from early data/operation

- Analysis tools are ready to take advantage of muons from collisions, when enough statistics is collected

\begin{tabular}{|c|c|c|}
\hline$\overbrace{\text { INFN }}$ & $\begin{array}{l}\text { G.Cattani - giordano.cattani@cern.ch } \\
\text { University of Rome "Tor Vergata" \& INFN Roma } 2\end{array}$ & $\begin{array}{r}\text { February 9, } 2010 \text { / Page } 20 \\
\text { X Workshop on Resistive Plate } \\
\text { Chambers and related detectors }\end{array}$ \\
\hline
\end{tabular}




\section{A muon collision candidate}

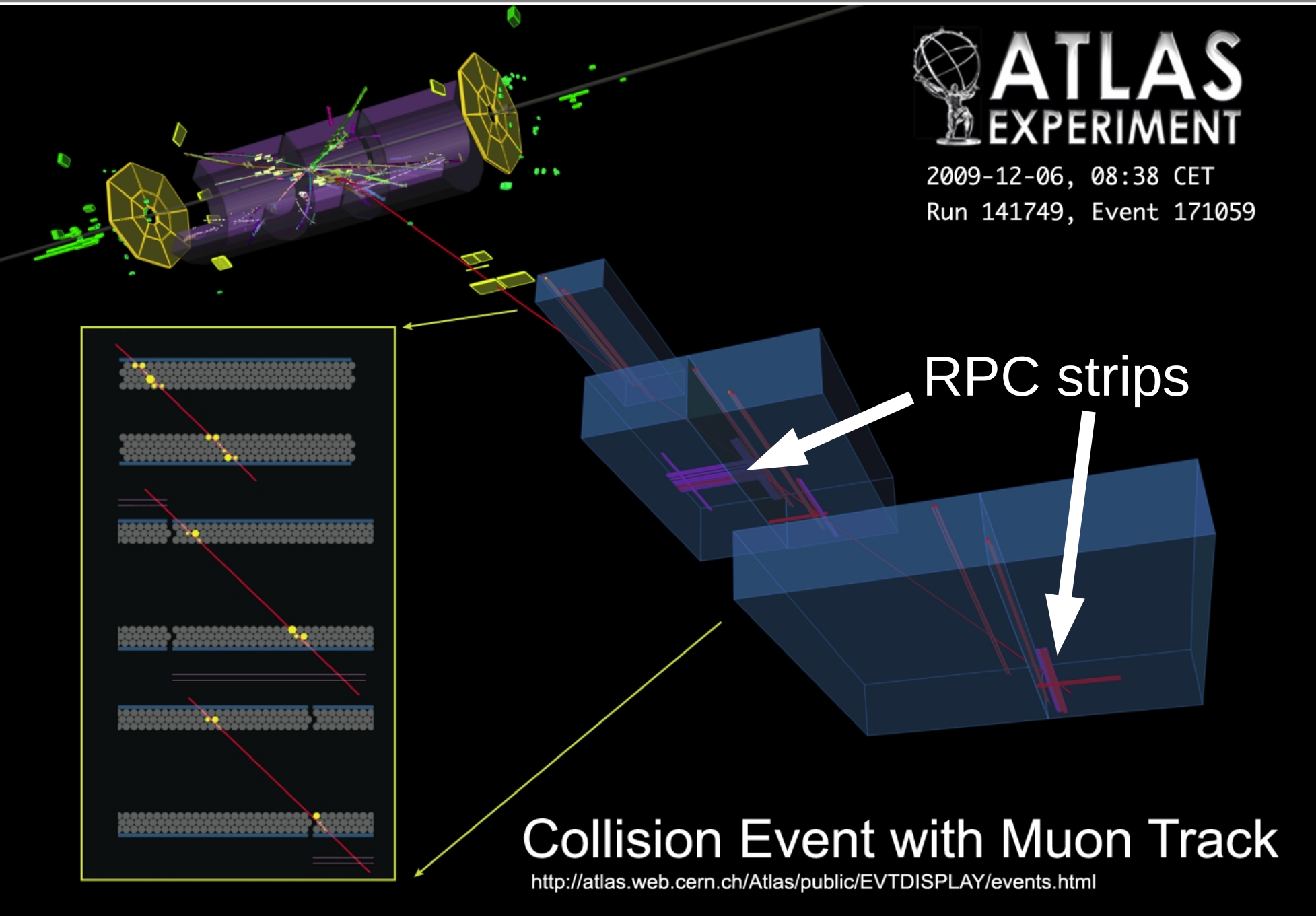

Chambers and related detectors 


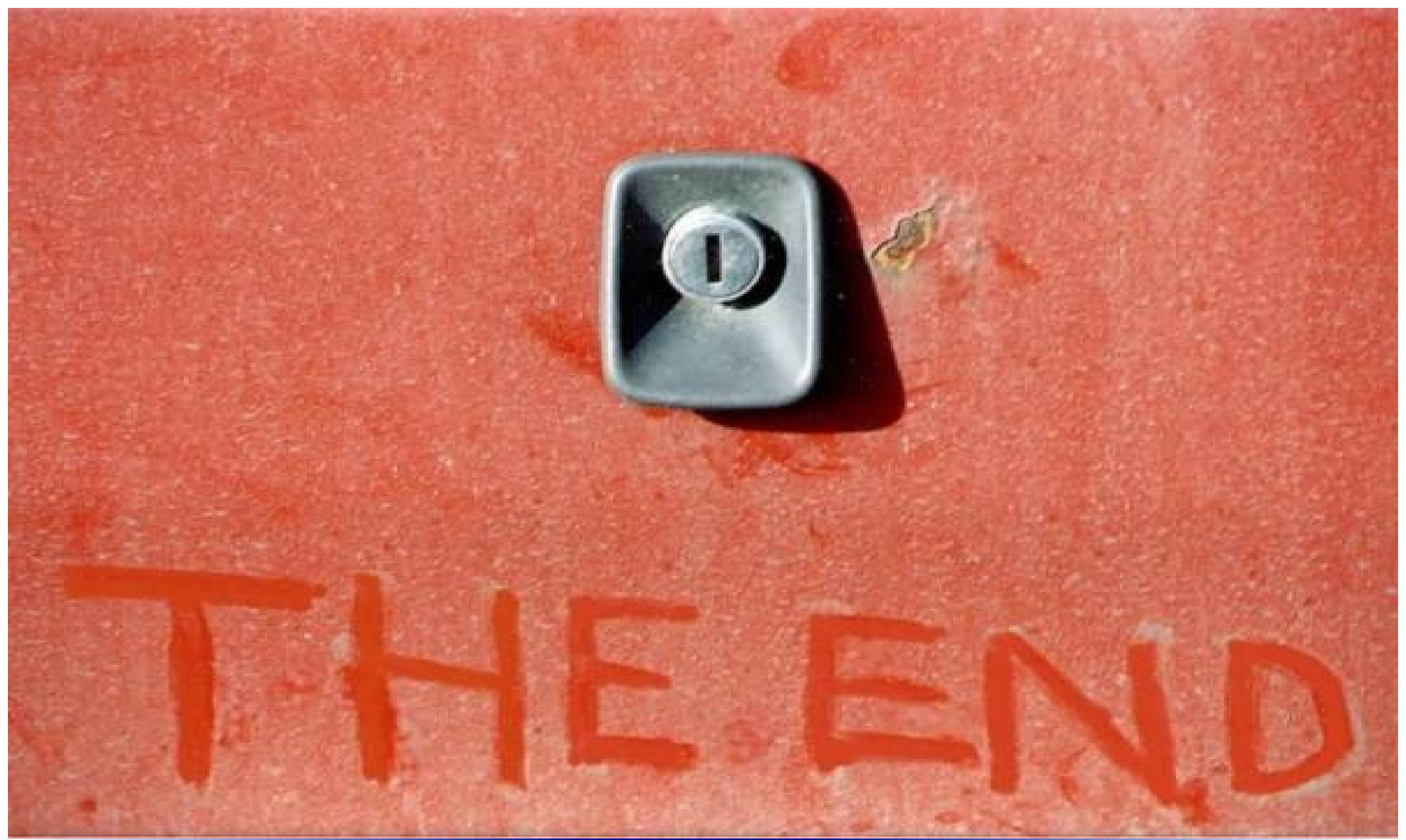




\section{Backup slides}

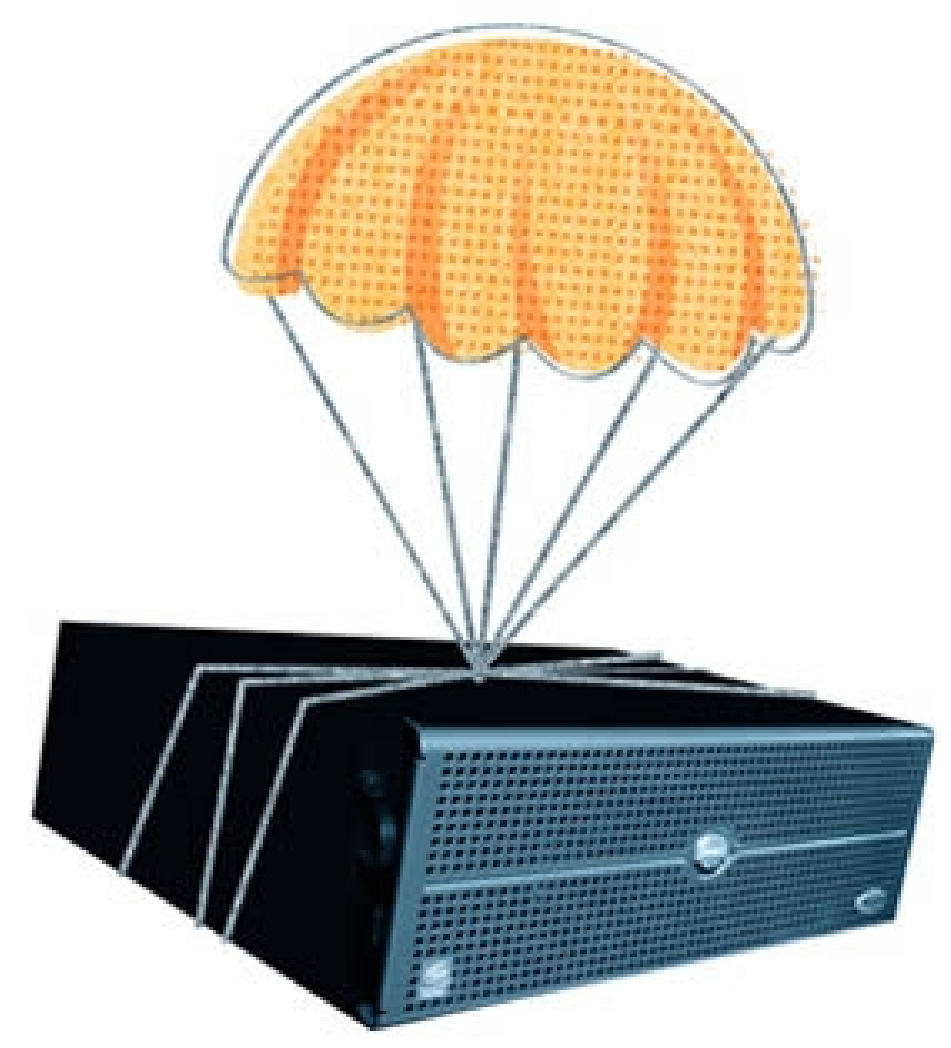

February 9, 2010 / Page 23

G.Cattani - giordano.cattani@cern.ch

INFN University of Rome "Tor Vergata" \& INFN Roma 2

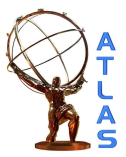

$X$ Workshop on Resistive Plate Chambers and related detectors 


\section{Muon Spectrometer: layout}

\begin{tabular}{|l|c|}
\hline Monitored drift tubes & MDT \\
- Coverage & $|\eta|<2.7$ (innermost layer: $|\eta|<2.0)$ \\
- Number of chambers & 1088 \\
- Number of channels & 339000 \\
- Function & Precision tracking \\
\hline Cathode strip chambers & CSC \\
- Coverage & $2.0<|\eta|<2.7$ \\
- Number of chambers & 32 \\
- Number of channels & 31000 \\
- Function & Precision tracking \\
\hline Resistive plate chambers & RPC \\
- Coverage & $|\eta|<1.05$ \\
- Number of chambers & 544 \\
- Number of channels & 359000 \\
- Function & Triggering, second coordinate \\
\hline Thin gap chambers & TGC \\
- Coverage & $1.05<|\eta|<2.7(2.4$ for triggering) \\
- Number of chambers & 3588 \\
- Number of channels & 318000 \\
- Function & Triggering, second coordinate \\
\hline
\end{tabular}

$-\quad$ The Muon Spectrometer of the ATLAS experiment is a very complex system:

- chambers of four different technologies are present (RPC, MDT, CSC, TGC)

- covers a very large area $\left(10.000 \mathrm{~m}^{2}\right)$

$\rightarrow \quad \sim 10^{6}$ read-out channels

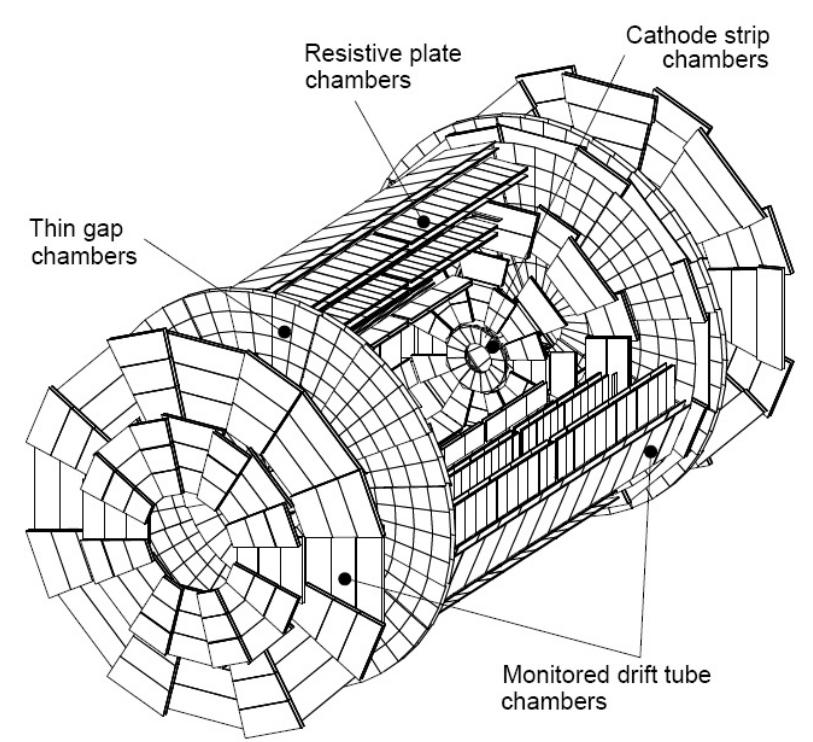




\section{Muon Spectrometer: momentum measurement}

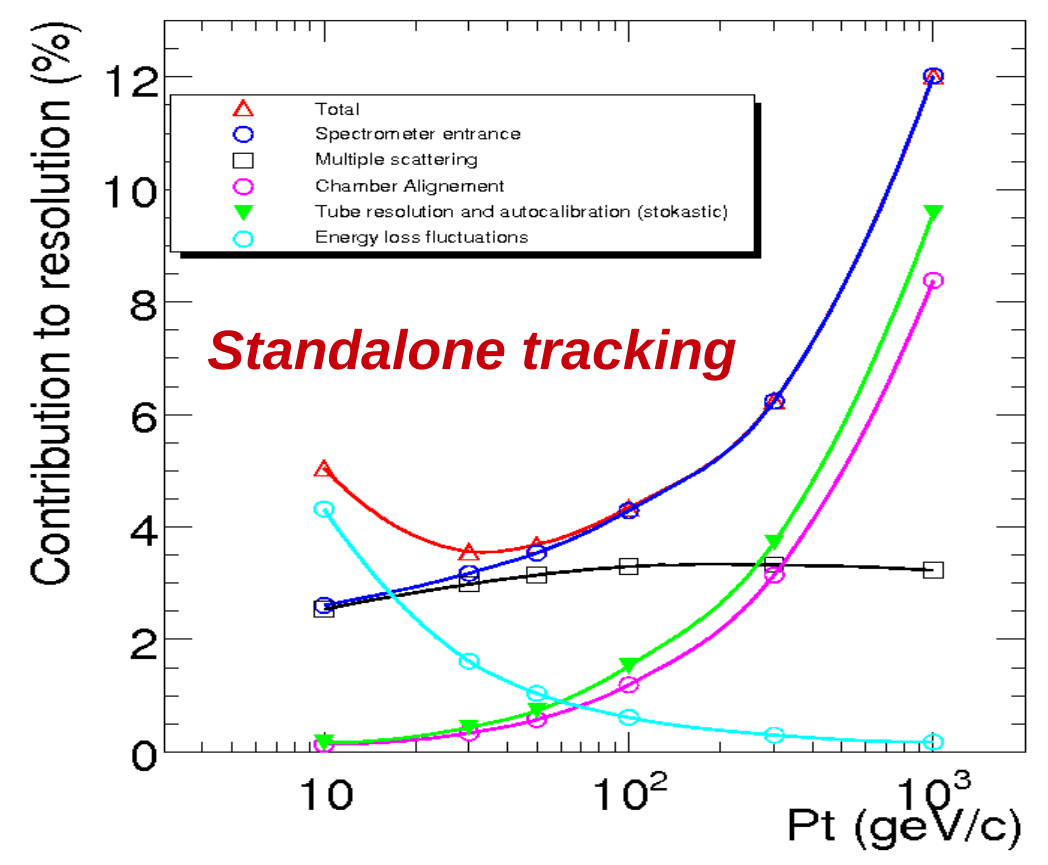

- Built to provide an excellent muon trigger and Bunch Crossing (BC) identification

- Achieve high precision momentum measurement

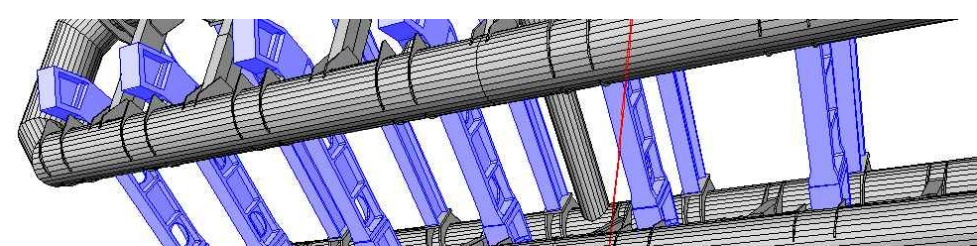

\begin{tabular}{|c|c|c|c|c|c|c|c|c|}
\cline { 3 - 9 } \multicolumn{2}{c}{} & \multicolumn{2}{c|}{ Chamber resolution (RMS) in } & Measurements/track & \multicolumn{2}{c|}{ Number of } \\
\hline Type & Function & $z / R$ & $\phi$ & time & barrel & end-cap & chambers & channels \\
MDT & tracking & $35 \mu \mathrm{m}(z)$ & - & - & 20 & 20 & $1088(1150)$ & $339 \mathrm{k}(354 \mathrm{k})$ \\
CSC & tracking & $40 \mu \mathrm{m}(R)$ & $5 \mathrm{~mm}$ & $7 \mathrm{~ns}$ & - & 4 & 32 & $30.7 \mathrm{k}$ \\
RPC & trigger & $10 \mathrm{~mm}(z)$ & $10 \mathrm{~mm}$ & $1.5 \mathrm{~ns}$ & 6 & - & $544(606)$ & $359 \mathrm{k}(373 \mathrm{k})$ \\
TGC & trigger & $2-6 \mathrm{~mm}(R)$ & $3-7 \mathrm{mn}$ & $4 \mathrm{~ns}$ & - & 9 & 3588 & $318 \mathrm{k}$ \\
\hline
\end{tabular}

February 9, 2010 / Page 25 


\section{Analysis strategy}

- In order to ensure redundancy/robustness, a twofold strategy is used for RPC detector studies

- Exploiting the precise tracking from the MDTs:

- bias on measurements due the trigger conditions is removed during off-line analysis

- extrapolation to RPC layers takes into account materials and magnetic field

- precise extrapolation allows to determine spatial resolution and to study small local effects

- applicable only to runs with MDTs on

- Using standalone tracking (see Angelo Guida's talk at this conference/session)

- does not depend on MDTs

- dedicated tracking algo avoids reconstruction bias on efficiency (by not using hits of a given layer)

- magnetic field/material effects not implemented yet

- extrapolation precision limited by RPC granularity 


\section{Hits \& clusters}

\section{Hits}

- The signal induced on a strip and read out by the front-end electronics is defined as a hit

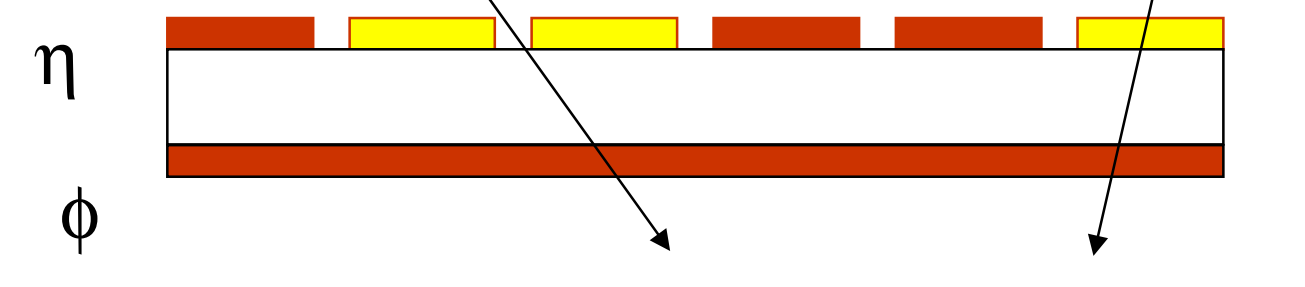

\section{Clusters}

A cluster is a group of hits from contiguous strips fulfilling the following rules:

- time difference between hits is < 15 ns

- time associated to a cluster is chosen as the minimum time of the hits in the cluster

- at each cluster is associated a "size" that is the number of hits in the cluster

- the position of the cluster is the centre of mass of the hits 


\section{Extrapolation of RPC triggered tracks to surface}

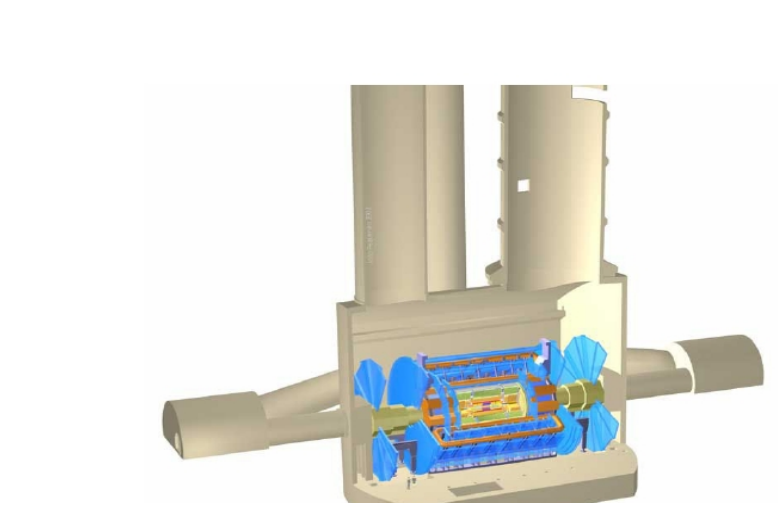

Sector 4
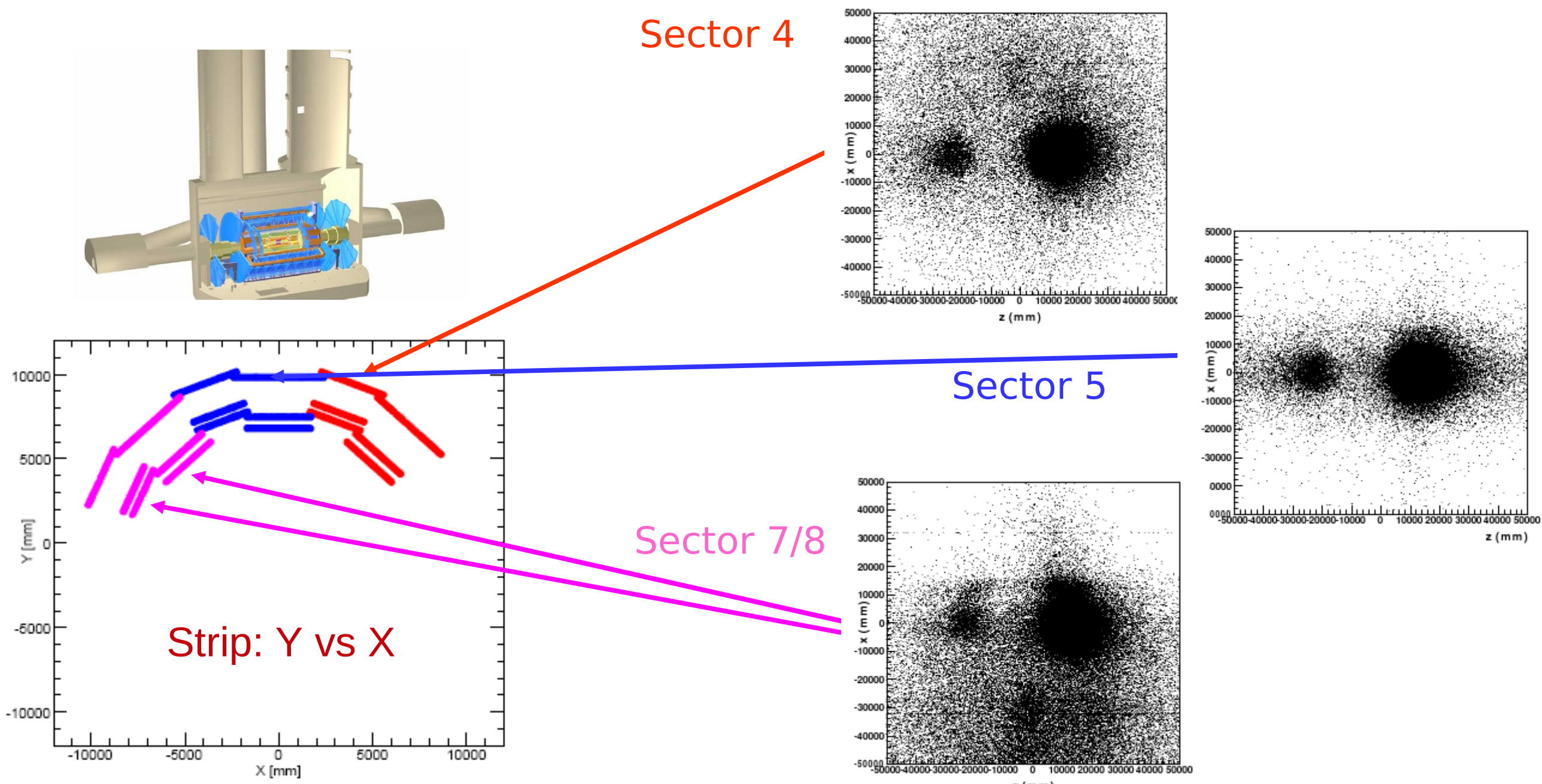